\title{
DNA Circuit System and Charge Transfer Mechanism
}

\author{
Kunming Xu \\ College of Ocean and Earth Sciences, Xiamen University, Xiamen, China \\ Email: kunmingx@xmu.edu.cn
}

Received 2013

\begin{abstract}
Based on the accurate identification of chemical structures as electric elements, we described charge transport mechanism in a DNA molecule by a stepwise $L C$ oscillatory circuitry, in which every base pair is a capacitor, every phosphate bridge is an inductor, and every deoxyribose is a charge router. The circuit model agrees with established experimental evidence that has supported super-exchange and hopping mechanisms so far. This alternative charge transport mechanism through both strands of DNA matches the fidelity and reliability of its chemical structure.
\end{abstract}

Keywords: Conductivity; Capacitor; Inductance; Oscillatory

\section{Introduction}

The electrical conductivity of a DNA molecule is of paramount important in biological function. It has been established that very fast charge transport can take place over a short distance $(\sim 37 \AA)$ and a slower transport may propagate over a long distance ( 200 $\AA$ ) [1-6]. The results have led to two hypotheses of charge transport mechanism in DNA, one indicating super-exchange, or tunnel, through the sugar phosphate bridge between the bound charge donor and acceptor for the short distance, and the other suggesting charge hopping between discrete bases through the DNA $\pi$-stack over long distance. Although both mechanisms are widely regarded, they are incompatible and ill-defined, giving the impression of loose or uncertain charge transfer along nucleotides. As genetic substance, DNA must possess well-defined electrical behavior. Here we show a DNA double helix as a stepwise $L C$ oscillatory circuitry, in line with the experimental evidence. Electrical harmonic oscillations have significant biological implications.

\section{DNA Circuit System}

The circuit model regards each base pair as an electric capacitor because the base pair is composed of two heterocyclic amines placed at a semi-conductive distance, capable of storing opposite charges. The hydrogen bonds between the base pair deliver electric pulse but prohibit direct current between them, which is a typical characteristic of electric capacitors. In the double helix, the phosphate bridges are twisted physically like both strands of a rope under torsion. The wound phosphate bridge can be treated as an electric inductor, capable of storing energy as well. The rigid structures of the Watson-Crick parities and of the pentose sugars help in forcing the torsion onto the phosphate bridges mechanically. Charge transport through the strands, like electric current through a coiled wire, produces electric inductance. Hence DNA structure is a circuitry composed of multiple oscillatory $L C$ circuits (Figure 1).

The deoxyribose is a key electric element in the circuit, which serves as a switch at the juncture (Figure 2). It has

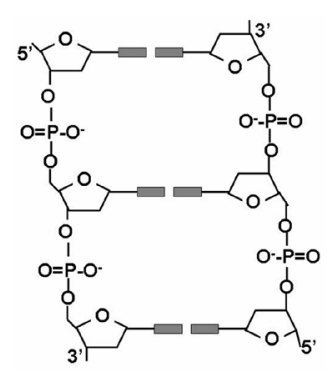

(a)

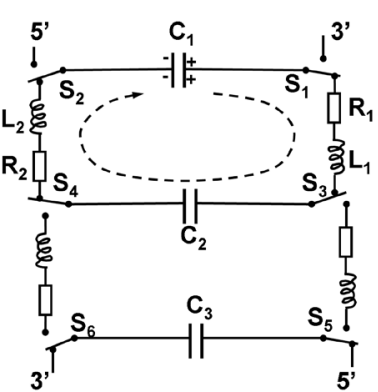

(b)

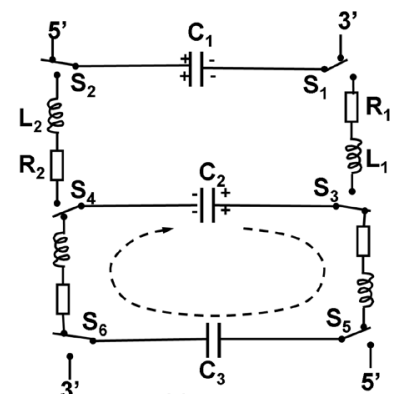

(c)

Figure 1. DNA double strands (a) along with stepwise oscillatory circuits (b) and (c), where the dashed arrows indicate electric current directions and the deoxyriboses are represented by electric switches. 


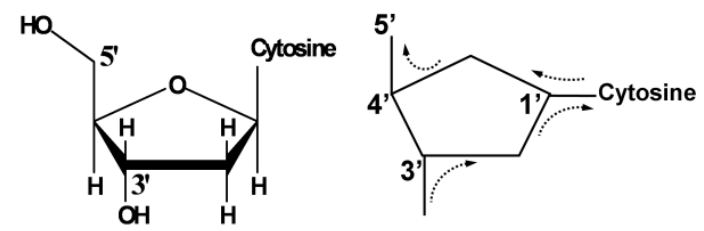

Figure 2. Electric currents directed by chiral carbons through a nucleoside. The dotted arrows represent electric current directions.

been stated that a chiral carbon center may transfer electrons towards a selective pathway due to its asymmetric polarizations [7]. Three chiral carbons in a deoxyribose direct electric charges in a selective course: C1' and C4' are levorotatory, and C3' is dextrorotatory [8]. Depending on whether the nucleotide base is positively charged or negatively charged, the switch connects C1' to C5' or connects C1' to C3' respectively. Direct charge flow between C3' and C4' is choked by both chiral carbons. The anti-parallel alignment of the pentose sugars on both strands determines that electric current forms a stepwise closed loop between every two adjacent base pairs.

Suppose in the closed circuit (Figure 1(b)), charges stored in capacitor $C_{1}$ transport through the phosphate inductors $L_{1}$ and $L_{2}$ to reach capacitor $C_{2}$ under the routing of the deoxyriboses. The anti-parallel alignment of the pentose sugars on both strands determines that positive charges transfer along the strand in the direction of $3^{\prime} \rightarrow$ 5' while negative charges transfer along the strand in the direction of $5^{\prime} \rightarrow 3$ '. Electric current forms a closed circuit so that by Kirchhoff's loop rule, we have voltage drop relationship of

$$
\frac{Q_{1}}{C_{1}}=I R_{1}+L_{1} \frac{d I}{d t}+\frac{Q_{2}}{C_{2}}+I R_{2}+L_{2} \frac{d I}{d t},
$$

where $I$ is electric current around the circuit unit, $Q_{1}$ and $Q_{2}$ are charges stored in base pair capacitances $C_{1}$ and $C_{2}$ respectively, and $R_{1}$ and $R_{2}$ are representative resistors along the path. Let

$$
\begin{gathered}
R_{1}+R_{2}=R, \\
L_{1}+L_{2}=L, \\
\frac{1}{C_{1}}+\frac{1}{C_{2}}=\frac{1}{C}, \\
Q_{1}+Q_{2}=Q_{0},
\end{gathered}
$$

then upon differentiation on both sides, Equation (1) becomes

$$
L \frac{\mathrm{d}^{2} I}{\mathrm{~d} t^{2}}+R \frac{\mathrm{d} I}{\mathrm{~d} t}+\frac{I}{C}=0 .
$$

This second-order differential equation is a damped harmonic oscillator. Depending on the relative values of $L, R$ and $C$, the system may be over-damped, critically damped, under-damped, or simple harmonic. When $R^{2}<4 L / C$, the circuit property falls into the two latter categories with a current function of

$$
I=I_{0} e^{-\frac{R}{2 L} t} \cos (\omega t),
$$

where $I_{0}$ is the initial current and $\omega$ is angular velocity of the oscillation with a value of

$$
\omega=\sqrt{\frac{1}{L C}-\frac{R^{2}}{4 L^{2}}} .
$$

The circuit system transfers electric charges from a base pair to another in stepwise oscillatory processes, i.e., from capacitor $C_{1}$ to $C_{2}$ (Figure 7.1b) and then from capacitor $C_{2}$ to $C_{3}$ (Figure 1(c)) along the double helix. Simple as it is, this model is in line with experimental evidence [1-6] so far established concerning DNA conductivity and reconciles both super-exchange and multi-step hopping mechanisms. The $L C$ circuit is robust and classical in physics, yet revolutionary in chemistry and biology. This interdisciplinary analysis produces a result of general interest in biological physics and may have potential influence in molecular electronics.

\section{Charge Transfer Mechanism}

It has been reported that the rate of electron transfer within a short distance decreases exponentially with increasing distance [2-5]. Such a phenomenon corresponds to the under-damped condition of the circuit system due to the relatively low $\left(R^{2}<4 L / C\right.$ ) resistance response of DNA backbones to the artificial introduction of voltage drop between base pairs in the experiments. However, when $R$ value is considerable, the exponential signal of electric current prevails over the sinusoidal cycle in Equation (7) and vanishes within a few oscillatory cycles. Since the distance between the base pairs of B-DNA is a fixed value of $3.4 \AA$ and each stepwise oscillatory cycle takes a certain interval of time for that distance, we may replace the time variable in Equation (7) with a distance variable $\Delta r$. In considering that electric current is a measure of electron transfer rate $k$, Equation (7) is equivalent to the Marcus correlation [9]

$$
k \propto e^{-\beta \Delta r}
$$

where $\beta$ values between 0.1 and $1.4 \AA^{-1}$ have been estimated for the double helix [2-5]. The dramatic difference can be ascribed to the uncertainty of resistance $R$ that is sensitive to various experimental conditions. The presence of considerable $R$ value along the circuit is because the phosphate bridges are under persistent high voltage drop induced artificially in experiments so that inductances partially manifest as resistances in the circuit. 
Based on frequency value of $10^{-10} \mathrm{~s}^{-1}$ measured by Fukui et al. [2], we simulate the under-damped oscillation with parameters of $C=0.02 \mathrm{pF}, L=0.01 \mu \mathrm{H}$, and $R$ $=100 \Omega$ in Equation (8). Assuming three radical cations are initially generated to trigger charge migration from a base pair to another through the stepwise oscillatory cycles, the current function of Equation (7) is calculated to be

$$
I=5.4 e^{-0.005 t} \cos (0.071 t),
$$

where electric current is in the unit of $\mathrm{nA}$ and time in ps (Figure 3). Suppose at $t=0$, capacitor $C_{1}$ carries charges in the polarity as shown in Figure 1(b), the deoxyribose switches $\left(S_{1}\right.$ to $\left.S_{4}\right)$ will route the charges in the dotted arrow direction. After one oscillatory cycle at $t=90 \mathrm{ps,}$ the charges reach capacitor $C_{2}$ so that switches $S_{3}$ and $S_{4}$ change their connections. The charges at capacitor $C_{2}$ will then be transferred to capacitor $C_{3}$ in the next step (Figure 1(c)). The amplitude of the electric current along the double helix decreases exponentially with each oscillatory cycle in the under-damped situation (Figure 3). Because each oscillatory cycle takes 90ps for charges to migrate $3.4 \AA$ along DNA strands, the $\beta$ value in Equation (9) is $0.13 \AA^{-1}$ in this case.

In vivo, we believe that natural electron transport from a base pair to another should incur trivial electric resistance. Even if there is certain electric resistance along the strands, the thermal energy produced by resistors would immediately be absorbed by both energy storage components of the base pair capacitor and the phosphate inductor. Hence electric resistance can be neglected. Let $C$ $=0.02 \mathrm{pF}, L=0.05 \mu \mathrm{H}$, and $R=5 \Omega$, the circuit declines into a series of $L C$ oscillators that transfer charges step by step along the strands harmonically at a slower pace. The frequency of the harmonic oscillation is slower than that of under-damped oscillation as can be predicted from $\omega$ formula under the relatively high value of inductance and trivial resistance. It takes about 200 ps for electrons to transfer from a base pair to the next. But the amplitude of the electric current remains almost the same in each oscillatory cycle. A comparison of under-damped oscillation and simple harmonic oscillation can be found in Figure 3.

In the stepwise $L C$ circuits, electric current is defined as positive when a base pair capacitor is being charged and negative when discharged in the next cycle down the chain. The stepwise oscillations agree with the evidence that has supported hopping mechanism through the base $\pi$-stack [1-6], but electric current through the sugar phosphate bridges is more reliable than the haphazard migration by hopping across the base rungs. Charges stored in capacitor $C_{1}$ transport through both strands to capacitor $C_{2}$, and will continue to move towards $C_{3}$ in the similar sinusoidal manner but at a lagging phase of $\pi$ in the cycle. During the processes, positive holes move in the

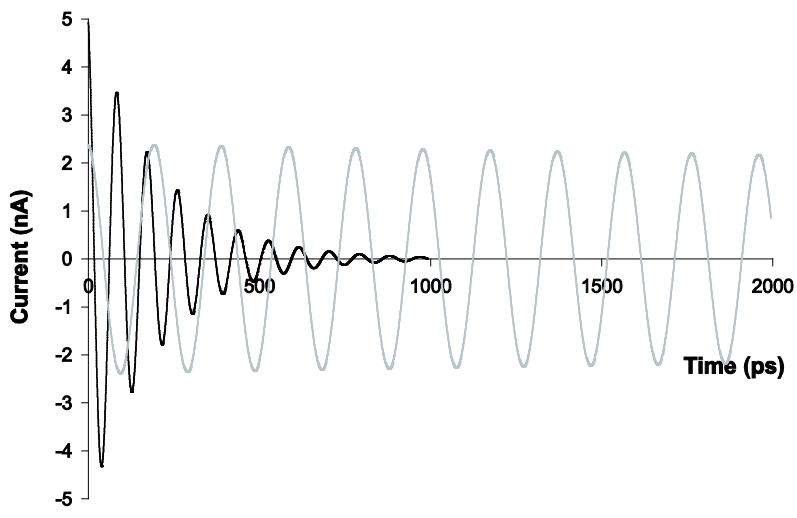

Figure 3. Model predictions for charge transfer of DNA circuit by fast stepwise under-damped oscillations in vitro (black curve) in contrast to slower stepwise simple harmonic oscillations in vivo (gray curve) where each cycle spans $3.4 \AA$ in distance. Charges undergoing under-damped oscillations have a hasty speed but cannot reach a long distance.

direction of 3' $\rightarrow$ 5' on one strand while electrons flow in the direction of $5^{\prime} \rightarrow 3^{\prime}$ on the other strand in good synchronization. The mechanisms for charge transport through long and short distances are the same. It takes more cycles of oscillations for charges to transport through longer sequences of base pairs. Without considering the effect of sequence dependence, traveling time is proportional to distance.

From Equation (8), we know that $\omega$ is codetermined by inductance $L$ and capacitance $C$ when $R$ is negligible. Assuming constant inductance for all nucleotides, the harmonic frequency would be determined by two neighboring capacitance values in the closed circuit, such as $C_{1}$ and $C_{2}$, which are specific to the base pairs. And this is perhaps the most subtle part of the story for it indicates that charge transfer rate is sequence dependent $[3-5,10]$. Because nucleotide bases have ionization potentials in the order of $\mathrm{G}<\mathrm{A}<\mathrm{C}<\mathrm{T}$ and electron affinities in the order of $\mathrm{C}<\mathrm{T}<\mathrm{G}<\mathrm{A}$ (disregarding negative sign) [11], it takes the least amount of energy to charge G:C base pair in the polarity of $+\mathrm{G}: \mathrm{C}-$ and requires the highest amount of energy to charge $+\mathrm{T}$ :A- capacitor. This means that the capacitances of the base pairs are in the order of $+\mathrm{G}: \mathrm{C}->+\mathrm{A}: \mathrm{T}->+\mathrm{C}: \mathrm{G}->+\mathrm{T}: \mathrm{A}-$ polarities. Thus there are four distinct capacitance values depending on the base pair and polarity.

Since $\omega$ is determined by two neighboring capacitances in series, it may take eight possible values. It is predicted that charge transport along the strands will produce various frequencies reflecting the identity of the bases. In other words, the pattern of charge transport is precisely controlled by the gene sequence. The +G:Ccapacitor has a higher capacitance than other base pairs and carries higher amount of charges in experiments whereas $+\mathrm{T}: \mathrm{A}-$ has a lower capacitance than other base pairs 
and is the limiting step or bottleneck in charge transfer along the DNA strands $[6,9]$. However, at long $(+\mathrm{T}: \mathrm{A}-)_{\mathrm{n}}$ sequences the size of the bottleneck remains the same so that the sequence distance dependence vanishes [9]. Because $+\mathrm{G}$ : $\mathrm{C}-$ has a relatively high capacitance, it is easy to trap charges, so it is likely to become the end point of a charge transport $[6,9]$. In this regard, the circuit model prediction agrees with experimental results completely.

Oscillatory current through the double helix is likely to have physiological significance. For example, if a base pair is mismatched at a certain position, then the oscillatory rhythms would be broken. Proofreading enzyme that scans the DNA sequence continually might easily locate the trouble point by the abnormal electric signal. Furthermore, biomolecules are inherently unstable. Only constant flow of energy prevents them from being disorganized. It stands to reason that the incessant charge vibrations in the genetic substance are vital for living organisms to maintain the integrity of the gene sequence.

From organic chemistry perspective, the base pair is capable of storing considerable amount of charges in either polarity by at least two conceivable mechanisms. First, both pyrimidine and purine are heterocyclic rings composed of carbon and nitrogen atoms. On the one hand, nitrogen is more electronegative than carbon for attracting higher electron density in covalent bonds with carbon. On the other hand, nitrogen atom has lone pair electrons to share with carbon under electron deficiency. The combination imparts great flexibility to the nucleotide bases for either holding or releasing electrons. Second, the heterocyclic rings are aromatic that possess diamagnetic ring current. In the circuit, aromatic ring current may reduce the charge saturation of the nitrogenous base through electromagnetic effect, and as a result increase the electric capacitance of the base pair. Both properties enable a base pair to be a good bipolar capacitor. The recognition of a base pair as a valid capacitor provides a sharp insight into molecular electronics.

A base pair is an electric capacitor. Alternate current passes through the hydrogen bonds. There are oscillating waves between each pair of nitrogenous bases within DNA nucleotides. Hydrogen bonding is a dynamic electronic action that delivers messages between the two bases, helping to balance the strain in a DNA double helix. Hydrogen bonds serve as the gateways to deliver messages. In this way, we describe the hydrogen bonds as a portal between the pair of bases. This dynamic interpretation is contrary to traditional static model of hydrogen bonds. Since the expression of any forces requires message exchanges, it is the wave information conveyed between pairs of bases that makes hydrogen bonds strong enough to hold the double helix of DNA together. Without the wave message, the atoms on both sides of hydrogen bonds would be detached and the organization of
DNA would start to unravel.

In the stepwise oscillatory circuit, a capacitor must be charged up to a threshold potential for positive charges to overflow through the ether bond of the deoxyribose. And this is mediated by the ether bond shown in Figure $\mathbf{4}$ as an electric switch. The oxygen atom would preferably use a $2 p_{x}$ and a $2 p_{y}$ orbitals to form covalent bonds with carbon atoms in the ether bridge. A full electron octet is a stable configuration of the oxygen atom because eight outer electrons are in a complete circulating cycle [8]. Electric current through the oxygen atom must comply with the circulation direction (Figure 4). Like a gasfilled tube, once a path is channeled by a positive threshold potential, the ether switch will remain on until the base pair is over-discharged to apply a negative potential of the same magnitude to the ether bond, thereby flipping the oxygen state from Figure $\mathbf{3}(\mathrm{ON})$ to $\mathbf{3}(\mathrm{OFF})$. To open the channel again requires the base pair to be recharged up to the initial threshold potential to flip the state over again. In the ON state, C1'-O covalent bond is $2 \mathrm{p}_{\mathrm{y}}-2 \mathrm{p}_{\mathrm{y}}$ overlap while $\mathrm{C} 4{ }^{\prime}-\mathrm{O}$ covalent bond is $2 \mathrm{p}_{\mathrm{x}}{ }^{-}$ $2 \mathrm{p}_{\mathrm{x}}$ overlap; in the OFF state, the nature of the covalent bonds is reverse (Figure 4). The chirality of C3', C1' and C4' determines that electric current is unidirectional through the ether bond and the phosphodiester bonds while the base pair is being charged and discharged cyclically. Such a mechanism ensures that the oscillatory current is in strict stepwise process. It has been found that charge migration in DNA is an ion-gated transport depending on the hydrated counter-ions and configurations [12]. But we believe that in vivo the circuit is gated by the dependable ether bond instead of by random ions coming from the environment.

Finally, we wish to present a general molecular model for phosphate in DNA backbone based on the electron circulations within oxygen atoms. The central phosphorus atom is bound by four oxygen atoms whose electron circulations are linked together forming four loops like a coiled wire, each loop involving an electron octet (Figure 5). Electric current passing through the molecule

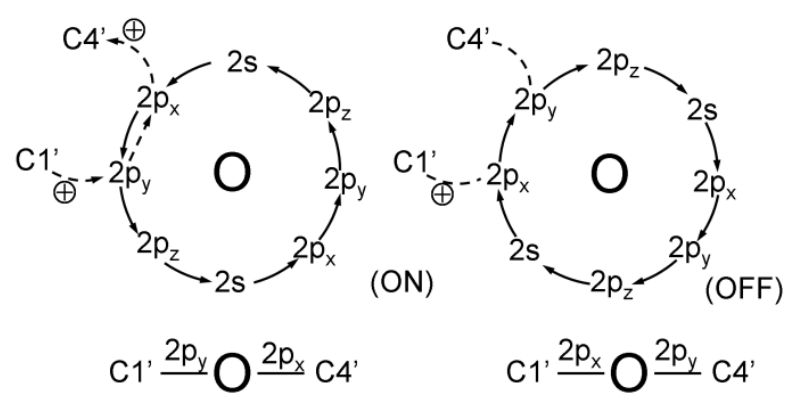

Figure 4. Atomic switch to control the flow of positive charges (dashed arrow) through the ether bond by flipping between two interconvertible states of contrary electron circulation (solid arrows) within the oxygen atom. 
<smiles>O=C(OP(=O)(O)OC(F)(F)F)c1ccccc1-c1ccccc1-c1ccccc1</smiles>

Figure 5. Schematic diagram of electron flow via the concatenated electron circulations within a phosphate bridge for electric induction corresponding to its chemical structure.

inevitably incurs considerable induction due to the convoluted electron flow, which explains why phosphate is an inductor and perhaps why ATP can serve as a physiological energy source when broken into ADP $+\mathrm{P}_{\mathrm{i}}$ even though the energy of the phosphoanhydride bond is not so large. The convolution collapse is the key factor in releasing a large amount of inductive energy. In DNA the electrical tension of phosphate chains must equilibrate with the mechanical torsion of the strands.

\section{REFERENCES}

[1] D. B. Hall, Holmlin, R. E., Barton and J. K. Oxidative, "DNA Damage through Long-Range Electron Transfer," Nature, Vol. 382, 1996, pp. 731-735. http://dx.doi.org/10.1038/382731a0

[2] K. Fukui, K. Tanaka, M. Fujitsuka, A. Watanabe and O. Ito, "Distance Dependence of Electron Transfer in Acridine-Intercalated DNA,” Journal of Photochemistry and Photobiology B: Biology, Vol. 50, 1999, pp. 18-27. http://dx.doi.org/10.1016/S1011-1344(99)00063-9

[3] C. Wan, T. Fiebig, O. Schiemann, J. K. Barton and A. H. Zewail, "Femtosecond Direct Observation of Charge Transfer between Bases in DNA," Proceedings of the National Academy of Sciences of the USA, Vol. 97, 2000, pp. 1405214055. http://dx.doi.org/10.1073/pnas.250483297

[4] B. Giese, J. Amaudrut, A.-K. Köhler, M. Spormann and S. Wessely, "Direct Observation of Hole Transfer through DNA by Hopping between Adenine Bases and by Tunneling," Nature, Vol. 412, 2001, pp. 318-320. http://dx.doi.org/10.1038/35085542

[5] M. E. Nunez, D. B. Hall and J. K. Barton, "Long-Range Oxidative Damage to DNA: Effects of Distance and Sequence," Chemical Biology, Vol. 6, 1999, pp. 85-97. http://dx.doi.org/10.1016/S1074-5521(99)80005-2

[6] M. Bixon and J. Jortner, "Long-Range and Very LongRange Charge Transport in DNA,” Chemical Physics, Vol. 281, 2002, pp. 393-408. http://dx.doi.org/10.1016/S0301-0104(02)00495-0

[7] A. S. Garay, "On the Role of Molecular Chirality in Biological Electronic Transport and Luminescence,” Life Sciences, Vol. 10, 1971, pp. 1393-1398. http://dx.doi.org/10.1016/0024-3205(71)90348-1

[8] K. Xu, "The Law of Nature: Spherical Quantity in Dynamic Calculus," Nova Scientific Publishers, Inc., New York, 2011, pp. 1-261.

[9] B. Giese, "Electron Transfer in DNA," Current Opinion in Chemical Biology, Vol. 6, No. 5, 2002, pp. 612-618. http://dx.doi.org/10.1016/S1367-5931(02)00364-2

[10] C. Nogues, S. R. Cohen, S. Daube, N. Apter and R. Naaman, "Sequence Dependence of Charge Transport Properties of DNA,” Journal of Physical Chemistry B, Vol. 110, 2006, pp. 8910-8913. http://dx.doi.org/10.1021/jp060870o

[11] S. D. Wetmore, R. J. Boyd and L. A. Eriksson, "Electron Affinities and Ionization Potentials of Nucleotide Bases," Chemical Physics Letters, Vol. 322, 2000, pp. 129-135. http://dx.doi.org/10.1016/S0009-2614(00)00391-2

[12] R. N. Barnett, C. L. Cleveland, A. Joy, U. Landman and G. B. Schuster, "Charge Migration in DNA: Ion-Gated Transport,” Science, Vol. 294, 2001, pp. 567-574. http://dx.doi.org/10.1126/science.1062864 